\title{
Approximate Fourier Domain Expression for Bloch-Siegert Shift
}

\author{
Esra Abaci Turk, ${ }^{1,2}$ Yusuf Ziya Ider, ${ }^{2}$ Arif Sanli Ergun, ${ }^{3}$ and Ergin Atalar ${ }^{1,2 *}$
}

Purpose: In this study, a new simple Fourier domain-based analytical expression for the Bloch-Siegert (BS) shift-based $B_{1}$ mapping method is proposed to obtain $\left|B_{1}^{+}\right|$more accurately while using short BS pulse durations and small off-resonance frequencies.

Theory and Methods: A new simple analytical expression for the BS shift is derived by simplifying the Bloch equations. In this expression, the phase is calculated in terms of the Fourier transform of the radiofrequency pulse envelope, and thus making the off- and on-resonance effects more easily understandable. To verify the accuracy of the proposed expression, Bloch simulations and MR experiments are performed for the hard, Fermi, and Shinner-Le Roux pulse shapes.

Results: Analyses of the BS phase shift-based $B_{1}$ mapping method in terms of radiofrequency pulse shape, pulse duration, and off-resonance frequency show that $\left|B_{1}^{+}\right|$can be obtained more accurately with the aid of this new expression. Conclusions: In this study, a new simple frequency domain analytical expression is proposed for the BS shift. Using this expression, $\left|B_{1}^{+}\right|$values can be predicted from the phase data using the frequency spectrum of the radiofrequency pulse. This method works well even for short pulse durations and small offset frequencies. Magn Reson Med 73:117-125, 2015. () 2014 Wiley Periodicals, Inc.

Key words: $B_{1}$ mapping; Bloch-Siegert shift; magnetic resonance imaging

\section{INTRODUCTION}

The Bloch-Siegert (BS)-based $B_{1}$ mapping technique was proposed by Sacolick et al. (1) as a phase-based $B_{1}$ mapping technique. This technique utilizes the fact that applying an off-resonance radiofrequency (RF) pulse after an excitation RF pulse adds phase to the excited spins; for a large off-resonance frequency, the added phase is directly proportional to the square of the $B_{1}$ field magnitude (2). This technique is insensitive to the spin relaxation, repetition time (TR), starting flip angle,

${ }^{1}$ National Magnetic Resonance Research Center (UMRAM), Bilkent University, Bilkent, Ankara, Turkey.

${ }^{2}$ Department of Electrical and Electronics Engineering, Bilkent University, Ankara, Turkey.

${ }^{3}$ Department of Electrical and Electronics Engineering, TOBB-University of Economics and Technology, Ankara, Turkey.

*Correspondence to: Ergin Atalar, Ph.D., UMRAM Cyberplaza, Block C, 2nd Floor, Cyberpark, Bilkent University, Bilkent, Ankara 06800, Turkey.

E-mail: ergin@ee.bilkent.edu.tr

Received 21 December 2012; revised 12 October 2013; accepted 13 October 2013

DOI 10.1002/mrm.25104

Published online 29 January 2014 in Wiley Online Library (wileyonlinelibrary. com).

(c) 2014 Wiley Periodicals, Inc. chemical shift, and $B_{0}$ field inhomogeneities. However, this technique has some limitations. For example, the sequence has a long echo time (TE) compared to that of a standard sequence without BS pulses. Furthermore, the sequence causes a high specific absorption rate due to the relatively long off-resonance RF pulse used to create the BS phase shift.

To improve this technique, there have been several studies on the optimization of the sequence and the off-resonance RF pulse shape (3-9). In Refs. 3 and 4, the optimization of the BS pulse shape was proposed to decrease the TE and specific absorption rate values. In both studies, better phase sensitivity was obtained in a shorter time and with lower onresonance excitation with designed pulses than the Fermi pulse. Specifically, an adiabatic RF pulse design was introduced to increase the sensitivity of the $\left|B_{1}\right|$ measurement in Ref. 5. Differently, to improve the sensitivity of the BS-based $B_{1}$ mapping method, reducing the off-resonance frequency was proposed in Ref. 6. In Refs. 7 and 8, a new sequence that caused a lower specific absorption rate than that of a spin echo sequence was proposed. In Ref. 9, a faster acquisition of the $B_{1}$ information and a minimized signal loss due to $\mathrm{T}_{2}^{*}$ effects were achieved. In both Refs. 3 and 6, the authors also mentioned that crusher gradients were added before and after a BS pulse to minimize the artifacts due to on-resonance excitation by the BS pulse. All of these studies improved the weaknesses of the BS-based $B_{1}$ mapping technique by modifying the sequence or the RF pulse shape.

In this study, our aim is to describe the parameters that affect the BS-based $B_{1}$ mapping method and to investigate the relationship between the effects of the off-resonance frequency, the RF pulse shape, and the duration of the RF pulse. To this end, we propose a general expression based on theoretical modeling that relates the Fourier transform of the off-resonance BS RF pulse envelope to the phase shift. To verify the accuracy of the proposed expression, we conducted extensive simulations and experiments. These simulations and experiments show that the proposed frequency domain expression is more accurate than the time domain expression that was proposed by the authors of the BS shift-based $B_{1}$ mapping method (1).

\section{THEORY}

In the BS phase shift-based $B_{1}$ mapping method, an offresonance $\mathrm{RF}$ pulse is applied after an excitation RF pulse to add a phase shift to the excited spins. The amount of phase shift $\left(\phi_{\mathrm{BS}}\right)$ depends on the applied RF field $\left[B_{1}^{+}(t)\right]$, the duration of the RF pulse (T), and the offset frequency between the RF pulse $\left[\omega_{R F}(t)\right]$ and the 
resonance frequency $\left(\omega_{0}\right)(2,10)$. In Ref. (1), it was shown that if $\omega_{\mathrm{RF}}(t)$ is much higher than $\left|\omega_{1}(t)\right|=\gamma\left|B_{1}^{+}(t)\right|$, where $\gamma$ is the gyromagnetic ratio, then in the $\omega_{0}$ rotating frame, the phase shift is directly related to the time integral of the square of $\left|\omega_{1}(t)\right|$, as given in Eq. [1]:

$$
\phi_{\mathrm{BS}} \approx \phi_{\mathrm{TD}}=\int_{0}^{T} \omega_{\mathrm{TD}}(t) \mathrm{d} t .
$$

where $\omega_{\mathrm{TD}}(t)=\frac{\left|\omega_{1}(t)\right|^{2}}{2 \omega_{\mathrm{RF}}(t)}$ and $\phi_{\mathrm{TD}}$ is the phase shift as the time domain approximation for the BS shift.

Long BS pulse durations cause long TE values, which result in signal loss due to the $\mathrm{T}_{2}^{*}$ and $\mathrm{T}_{2}$ effects; therefore, the use of a small pulse duration becomes important. However, as our preliminary results have shown (11) for small pulse durations, there is a significant difference between the actual phase shift $\left(\phi_{\mathrm{BS}}\right)$, as obtained by the solution of the complete Bloch equations, and the phase shift given by Eq. [1]. These results are obtained, when the pulse duration is changed while keeping the same peak $\left|B_{1}\right|$ value for each pulse duration, even if the condition $\omega_{\mathrm{RF}}(t)>>\left|\omega_{1}(t)\right|$ is satisfied. This difference $\left(\phi_{\text {res }}\right)$ is defined as:

$$
\phi_{\mathrm{res}}=\phi_{\mathrm{BS}}-\phi_{\mathrm{TD}} .
$$

In fact, $\phi_{\text {res }}$ can also be defined as the phase accumulation in the $\omega_{0}+\omega_{\mathrm{TD}}(t)$ rotating frame. Consequently, to obtain $\phi_{\text {res }}$, the Bloch equations are solved in this rotating frame. (Note that this rotating frame is named the BS time domain (BSTD) rotating frame.) In the BSTD rotating frame, $B_{1}^{+}(t)$ is defined as:

$$
B_{1}^{+}(t)=B_{1}^{\mathrm{e}}(t) \exp \left[i\left(\int_{0}^{t}\left(\omega_{\mathrm{RF}}(\tau)-\omega_{\mathrm{TD}}(\tau)\right) \mathrm{d} \tau+\theta+\theta_{0}\right)\right],
$$

where $B_{1}^{\mathrm{e}}(t)$ is the envelope, $\theta$ is the phase of the applied BS shift RF pulse, and $\theta_{0}$ is the accumulated phase until the beginning of the BS pulse.

The Bloch equation in matrix form in the BSTD rotating frame is given as:

$$
\frac{\mathrm{d}}{\mathrm{d} t}\left(\begin{array}{c}
M_{X} \\
M_{\mathrm{y}} \\
M_{z}
\end{array}\right)=\left(\begin{array}{ccc}
0 & -\omega_{T D}(t) & -\omega_{1 y}(t) \\
\omega_{\mathrm{TD}}(t) & 0 & \omega_{1 x}(t) \\
\omega_{1 y}(t) & -\omega_{1 x}(t) & 0
\end{array}\right)\left(\begin{array}{c}
M_{X} \\
M_{y} \\
M_{z}
\end{array}\right)
$$

where $\omega_{1 x}(t)$ and $\omega_{1 y}(t)$ are the real and imaginary parts, respectively, of $\omega_{1}(t)$ as follows:

$$
\begin{aligned}
& \omega_{1 x}(t)=\gamma B_{1}^{\mathrm{e}}(t) \cos \left(\int_{0}^{t}\left(\omega_{\mathrm{RF}}(\tau)-\omega_{\mathrm{TD}}(\tau)\right) \mathrm{d} \tau+\theta+\theta_{0}\right), \\
& \omega_{1 y}(t)=\gamma B_{1}^{\mathrm{e}}(t) \sin \left(\int_{0}^{t}\left(\omega_{\mathrm{RF}}(\tau)-\omega_{\mathrm{TD}}(\tau)\right) \mathrm{d} \tau+\theta+\theta_{0}\right) .
\end{aligned}
$$

In the BSTD rotating frame, the magnetization vector at time zero (the time that BS RF pulse is started) is $\mathrm{M}(0)=\left(\begin{array}{lll}M_{0} & 0 & 0\end{array}\right)^{T}$, where $T$ stands for the vector transpose. Under this condition, the time derivative of $M_{X}$ is very small, and it is assumed that $M_{X}$ remains almost constant throughout the BS RF pulse. Therefore, the system of differential equations is reduced to:

$$
\frac{\mathrm{d}}{\mathrm{d} t}\left(\begin{array}{c}
M_{\mathrm{y}} \\
M_{\mathrm{z}}
\end{array}\right)=\left(\begin{array}{cc}
0 & \omega_{1 x}(t) \\
-\omega_{1 x}(t) & 0
\end{array}\right)\left(\begin{array}{l}
M_{y} \\
M_{z}
\end{array}\right)+\left(\begin{array}{c}
\omega_{\mathrm{TD}}(t) \\
\omega_{1 y}(t)
\end{array}\right) M_{0} .
$$

A complex $M_{\mathrm{yz}}$ variable is defined as $M_{\mathrm{yz}}=M_{\mathrm{y}}+i M_{\mathrm{z}}$. The solution for the $M_{y z}(T)$ variable for the pulse duration $T$ is found to be the following (Appendix A):

$$
M_{\mathrm{yz}}(T)=M_{0} \int_{0}^{T}\left(\omega_{\mathrm{TD}}(t)+i \omega_{1 y}(t)\right) \exp \left(-i \int_{t}^{T} \omega_{1 x}(s) \mathrm{d} s\right) \mathrm{d} t .
$$

To simplify the solution, the exponential term is simplified using the fact that $\omega_{\mathrm{RF}}(t)>>\left|\omega_{1}(t)\right|$ and using the following argument: $\omega_{1 x}(s)$ is the multiplication of a slowly varying function $B_{1}^{\mathrm{e}}(t)$ and a cosine function with a much higher frequency that changes slowly between $\omega_{\text {RFmin }}$ and $\omega_{\text {RFmax }} \cdot \int_{t}^{T} \omega_{1 x}(s) \mathrm{d} s$ becomes bounded by a maximum value, which is determined by $B_{1 \max }^{\mathrm{e}}$ times the integral of the cosine function during a half cycle of the minimum frequency $\omega_{\mathrm{RFmin}}$. In other words, this integral becomes bounded by $\frac{2 \gamma\left|B_{1 \max }^{e}\right|}{\omega_{\mathrm{RF}} \mid}$, which is much smaller than 1 for $\omega_{R F \min }>>\left|\omega_{1 \max }\right|$, where $\omega_{1 \max }=\gamma B_{1 \max }^{\mathrm{e}}$. (Note that for a hard pulse, $B_{1 \max }^{\mathrm{e}}$ is the magnitude of the RF pulse, and for Shinner-Le Roux (SLR) and Fermi pulses, $B_{1 \max }^{\mathrm{e}}$ corresponds to the peak values.).

$$
\exp \left(-i \int_{t}^{T} \omega_{1 x}(s) \mathrm{d} s\right) \approx 1-i \int_{t}^{T} \omega_{1 x}(s) \mathrm{d} s,
$$

With this simplification, the solution can be separated easily into its real and imaginary parts and the components $M_{y}$ and $M_{z}$ can be obtained as:

$$
\begin{aligned}
& M_{y}(T) \approx M_{0} \int_{0}^{T} \omega_{\mathrm{TD}}(t) \mathrm{d} t+M_{0} \int_{0}^{T} \int_{t}^{T} \omega_{1 y}(t) \omega_{1 x}(s) \mathrm{d} s \mathrm{~d} t \\
& M_{Z}(T) \approx M_{0} \int_{0}^{T} \omega_{1 y}(t) \mathrm{d} t-M_{0} \int_{0}^{T} \int_{t}^{T} \omega_{\mathrm{TD}}(t) \omega_{1 x}(s) \mathrm{d} s \mathrm{~d} t .
\end{aligned}
$$

Because we assume that $M_{X}(T)=M_{0}$ and $M_{Y}(T)$ are small, the phase can be found using $\phi=-\tan ^{-1} \frac{M_{y}(T)}{M_{0}} \approx-$ $\frac{M_{y}(T)}{M_{0}}$ (note that the minus sign is due to the fact that the phase is defined in the left-hand direction), and the expression for $\phi_{\text {res }}$ in the BSTD rotating frame becomes:

$$
\phi_{\mathrm{res}} \approx-\int_{0}^{T} \int_{t}^{T} \omega_{1 y}(t) \omega_{1 x}(s) \mathrm{d} s \mathrm{~d} t-\int_{0}^{T} \omega_{\mathrm{TD}}(t) \mathrm{d} t
$$

To find the phase shift defined in the $\omega_{0}$ rotating frame, which is the actual phase shift, we add the term $\phi_{\mathrm{TD}}$ to $\phi_{\mathrm{res}}$ as given in Eq. [2]. Note that the term $\theta_{0}$, which is the phase accumulated prior to the beginning of the BS pulse, is also subtracted to obtain the phase shift in the $\omega_{0}$ rotating frame:

$$
\phi_{\mathrm{BS}} \approx-\int_{0}^{T} \int_{t}^{T} \omega_{1 y}(t) \omega_{1 x}(s) \mathrm{d} s \mathrm{~d} t-\theta_{0}
$$

Because the contribution of $\theta_{0}$ is canceled using the difference of the two acquisitions taken with positive 

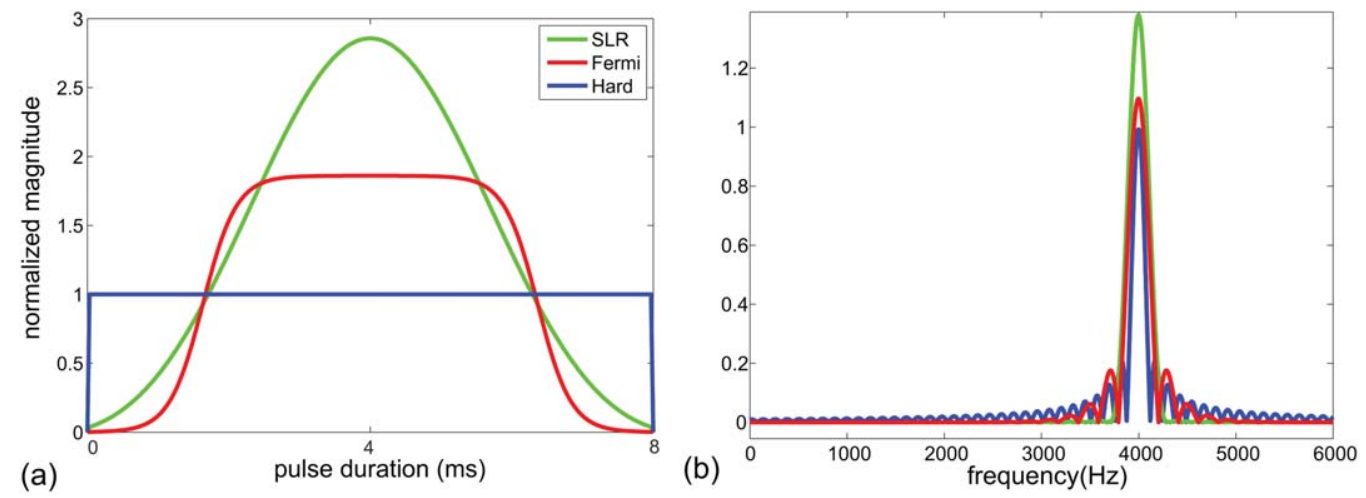

FIG. 1. a: Pulse shapes used in the analysis. b: Fourier transforms of each pulse with a 4-kHz offset frequency and an 8-ms pulse duration.

and negative offset frequencies, $\theta_{0}$ is ignored in the rest of the equations. Note that $\omega_{1 x}(s)$ and $\omega_{1 y}(t)$ remain at the same values as defined in the BSTD frame.

Using the Fourier transform of $\omega_{1}(t)$, which is denoted by $\Omega_{1}(f)$ (i.e., $\Omega_{1}(f)=\int_{-\infty}^{\infty} \omega_{1}(t) e^{-i 2 \pi f t} \mathrm{~d} t$ ), the final expression becomes:

$$
\phi_{\mathrm{BS}} \approx-\int_{-\infty}^{\infty} \frac{\left|\Omega_{1}(f)\right|^{2}}{4 \pi f} \mathrm{~d} f
$$

Note that the detailed derivation of this expression is given in Appendix B.

This expression is simplified using the Hilbert transform. The Hilbert transform of a function is defined as $\mathcal{H}\{g(t)\}=\frac{1}{\pi} \int_{-\infty}^{-\infty} \frac{g(\tau)}{t-\tau} \mathrm{d} \tau$. The Hilbert transform is defined as the Cauchy principal value of the integral in this equality whenever the value of the integral around the pole $t=\tau$ exists. The Cauchy principal value is obtained by considering a finite range of integration that is symmetric about the point of singularity and the region with the singularity is excluded. While the interval of the integral approaches $\infty$, the length of the excluded interval approaches zero. The Hilbert transform of $g(t)$ at $t=$ 0 can be expressed as $\left.\mathcal{H}\{g(t)\}\right|_{t=0}=-\frac{1}{\pi} \int_{-\infty}^{-\infty} \frac{g(\tau)}{\tau} \mathrm{d} \tau$. With this information, the Fourier domain approximation of the BS shift becomes the following:

$$
\phi_{\mathrm{BS}} \approx \phi_{\mathrm{FD}}=-\int_{-\infty}^{\infty} \frac{\left|\Omega_{1}(f)\right|^{2}}{4 \pi f} \mathrm{~d} f=\frac{\left.\mathcal{H}\left\{\left|\Omega_{1}\left(f^{\prime}\right)\right|^{2}\right\}\right|_{f^{\prime}=0}}{4}
$$

To find the peak of the $B_{1}$ field from the phase in the $\omega_{\mathrm{RF}}(t)>>\left|\omega_{1}(t)\right|$ region, Eq. [15] is changed to the following equation:

$$
B_{1 \text { peak }} \approx \frac{1}{\gamma} \sqrt{\frac{4 \phi_{\mathrm{FD}}}{\left.\mathcal{H}\left\{\left|\Omega_{\mathrm{norm}}\left(f^{\prime}\right)\right|^{2}\right\}\right|_{f^{\prime}=0}}}
$$

where $\Omega_{1}(f)=\gamma B_{1 \text { peak }} \Omega_{\text {norm }}(f)$.

As an example, Eq. 15 is analytically solved for a hard pulse with a pulse duration $(T)$ and constant offset frequency $\left(\omega_{\mathrm{RF}}\right)$ in $\omega_{\mathrm{RF}}(t)>>\left|\omega_{1}(t)\right|$. The resultant expression is as follows:

$$
\phi_{\mathrm{FD}}=\frac{\left(\gamma B_{1 \text { peak }}\right)^{2} T}{2\left(\omega_{\mathrm{RF}}\right)}\left[1-\sin c\left(\frac{\omega_{\mathrm{RF}}}{\pi} T\right)\right] .
$$

Analysis of this new approximated frequency domain BS relation (Eq. [15]) for the hard, Fermi, and SLR pulse shapes and a comparison of the results with (i) the solution of the time domain approximated relation (Eq. [1]), (ii) the results of the Bloch simulations, and (iii) the results of the experiments are given in the Results section.

\section{METHODS}

To investigate the parameters that affect the BS shiftbased $B_{1}$ mapping method and to verify Eq. [15], which is described in the theory section, Bloch simulations and MR experiments are performed for different pulse shapes. For the BS $B_{1}$ mapping method, properly choosing the off-resonance RF pulse shape is critical because this affects the phase value, the minimum offset frequency that can be used, and the minimum undesired magnetization tilting effect. In (1), the hard, Fermi, adiabatic hyperbolic secant, and the adiabatic tanh/tan pulses were compared in terms of their frequency range that contains $99 \%$ of spin excitation and the constant, $K_{\mathrm{BS}}$, describing the phase shift. As a result, the Fermi pulse was chosen for the experiments. In our experiments, however, only the hard, Fermi, and SLR pulse shapes are used. The envelope of the Fermi pulse is defined by the expression $\frac{1}{1+e^{\mid\left(|(t)|-t_{0}\right) / a}}$, where the parameters $t_{0}$ and $a$ are defined as $T=2 t_{0}+13.81 a$ and $t_{0}=10 a$, and $T$ is the pulse duration. The SLR pulse is designed with a $0.5 \%$ passband ripple, $1 \%$ reject ripple, and $0.3 \mathrm{kHz}$ bandwidth using the VESPA-RFPulse tool (12). In Figure 1, we present the pulse shapes and their frequency domain patterns. The pulse magnitudes are normalized in such a way that the same phase values can be obtained for an $8-\mathrm{ms}$ pulse duration and a $4-\mathrm{kHz}$ offset frequency.

The experiments were performed in a 3T scanner (MAGNETOM Trio a Tim System, Siemens Healthcare, Erlangen, Germany). During the experiments, a FLASH sequence that was modified by adding an off-resonance 


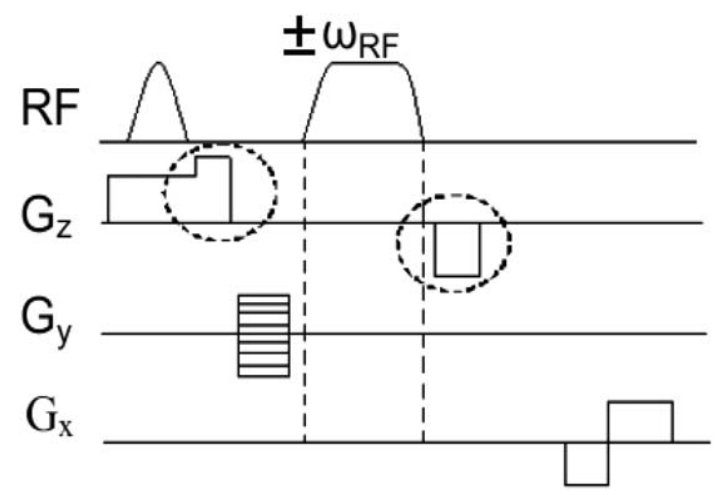

FIG. 2. Pulse sequence used in the experiments. Crusher gradients (encircled by a dotted line) are used to reduce the effect of the on-resonance excitation by the BS pulse.

pulse after the excitation RF was used. The excitation RF was a sinc pulse with a 1-ms duration. Crusher gradients with a 1-ms duration in the slice selection direction were added to the sequence before and after the offresonance pulse (6), and the phase encoding gradient was applied before the off-resonance RF pulse to avoid encoding the undesired off-slice spins that were excited by the off-resonance RF pulse. Figure 2 shows the modified sequence. In each experiment, two phase images were acquired using a BS pulse with positive and negative offset frequencies, and the phase shifts were calculated by taking the difference between these two phase images. For each experiment, the imaging parameters were set to $150-\mathrm{ms}$ TR, 5 -mm slice thickness, $256 \times 256$ in-plane resolution, and 200-mm field of view. The $\left|B_{1}^{+}\right|$ value, which is calculated by Eq. [1] using the phase shift obtained with a Fermi pulse with an 8-ms pulse duration and a $4-\mathrm{kHz}$ offset-frequency for a given $\mathrm{RF}$ voltage, is used to establish the calibration factor between the peak $\left|B_{1}^{+}\right|$and the applied RF voltage level. In the experiments, a cylindrical $1900 \mathrm{~mL}$ Siemens phantom with a $10-\mathrm{cm}$ diameter $(3.75 \%$ NISO $4 \times 6 \mathrm{H} 2 \mathrm{O}+5 \%$ $\mathrm{NaCl})$ was used and for the RF transmission and reception, a transmit/receive rectangular coil with $10 \times 23 \mathrm{~cm}$ dimension and tuned by eight capacitors was used, unless otherwise indicated. Note that the flip angle is space dependent due to the usage of the surface coil. Therefore, for each experiment, the data were collected from the same region with a maximum and constant $B_{1}^{+}$ field distribution.

For the simulations, the Bloch equations were solved numerically in MATLAB (Mathworks, Natick, MA) using rotation matrices in an $\omega_{0}$ rotation frame. The $M_{x}, M_{y}$, and $M_{z}$ magnetization components were described by 10 $\times 10$ matrices with the elements on the $x-y$ plane, and it was assumed that the elements of the matrices were located at a distance of $1.56 \mathrm{~mm}$ from each other on the $x-y$ plane. For initialization, the $M_{z}$ magnetization components were taken as one, and the $M_{x}$ and $M_{y}$ components were zero. Crusher gradients were also added to the simulations.

During the experiments and the simulations, when an extreme phase value, $\pi$ or $-\pi$, was reached, the $2 \pi$ discontinuity of the extracted phase appeared. To address this phase wrapping problem in the one-dimensional case, the "unwrap" function of MATLAB was used by assuming phase continuity, and in the two-dimensional case (for MR phase images), Goldstein's branch cut method was used (13). To correct for the effect of the $B_{0}$ offset frequency in the simulations and calculations, especially for small offset frequencies (i.e., $100 \mathrm{~Hz}$ ), $B_{0}$ maps were obtained using two gradient echo images with different TEs (i.e., $\Delta \mathrm{TE}=1 \mathrm{~ms}$ ), whereas the other imaging parameters were kept constant (i.e., $100 \mathrm{~ms}$ TR, $5 \mathrm{~mm}$ slice thickness, $256 \times 256$ in-plane resolution, and $200 \mathrm{~mm}$ field of view).

\section{Effect of the Pulse Duration}

While using experiments and simulations to investigate the effect of the pulse duration for the hard and Fermi pulse shapes, the pulse duration was varied between 150 $\mu \mathrm{s}$ and $2 \mathrm{~ms}$ with $50 \mu \mathrm{s}$ steps, and the SLR pulse shape duration was varied between $300 \mu$ s and 2 ms with 50 $\mu \mathrm{s}$ steps. The TE values are set according to the BS pulse from 6.5 to $8.5 \mathrm{~ms}$. The experiments were repeated seven times for each pulse and pulse duration. The pulse duration versus phase plots were computed with the mean values, and the standard deviations computed across the seven repeats. For each experiment, the offset frequency was set to $2 \mathrm{kHz}$. To generate a similar range of phase shifts for the hard, Fermi, and SLR pulse shapes, the applied RF voltage were adjusted and the peak $\left|B_{1}^{+}\right|$values were estimated to be $12.6 \mu \mathrm{T}$ for the hard pulse, $16.2 \mu \mathrm{T}$ for the Fermi pulse, and $21.1 \mu \mathrm{T}$ for the SLR pulse, where $\left(\omega_{1} / \omega_{R F}\right) \leq 0.5$.

To visualize the effect of the pulse duration for a specific case, a 16-cm diameter cylindrical phantom was prepared. Four small cylinders with 3.5-mm diameter were placed inside the cylindrical phantom. The small cylinders were filled with oil, and the outsides of the small cylinders were filled with water mixed with $0.2 \%$ $\mathrm{CuSO}_{4}$. During these experiments, a body coil was used for transmission and a 12-channel Siemens head coil was used for reception. As a BS pulse, a Fermi pulse with $0.6-\mathrm{ms}$ pulse duration and $2-\mathrm{kHz}$ offset frequency was used. The imaging parameters were set to $150-\mathrm{ms}$ TR, 5-mm slice thickness, $256 \times 256$ in-plane resolution, and $200-\mathrm{mm}$ field of view, $60^{\circ}$ flip angle, $7-\mathrm{ms}$ TE.

\section{Effect of the Off-Resonance Frequency}

In the BS shift-based $B_{1}$ mapping technique, the phase shift is inversely proportional to $\omega_{\mathrm{RF}}$, as indicated in Eq. [1]. To obtain a more accurate $\left|B_{1}^{+}\right|$estimate, one may prefer to decrease $\omega_{\mathrm{RF}}$. The maximum $\left|B_{1}^{+}\right|$value that can be calculated accurately is then limited by the requirement $\omega_{\mathrm{RF}}>>\left|\omega_{1}(t)\right|$. To understand the relation between the phase and the off-resonance frequency and to compare the results of frequency domain approximation (Eq. [15]) and time domain approximation (Eq. [1]), the results of the simulations and experiments for different offset frequencies were investigated. For this analysis, hard, Fermi, and SLR pulse shapes with 8-ms pulse durations were used. The TE value was set to $14.5 \mathrm{~ms}$ in these experiments. According to the reference, $\left|B_{1}^{+}\right|$value obtained with a Fermi pulse with an 8 -ms pulse duration 


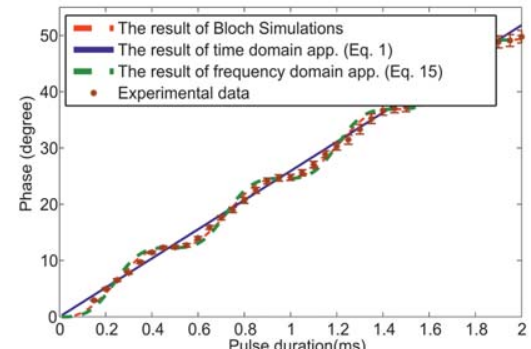

(a)

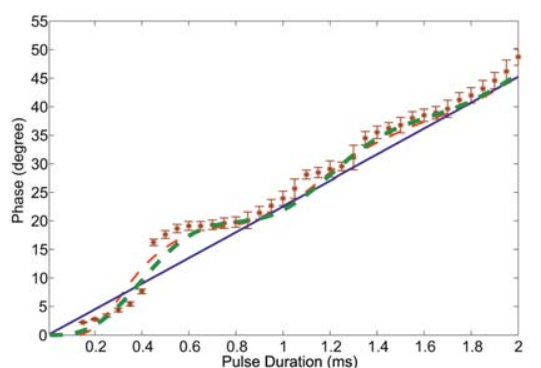

(b)

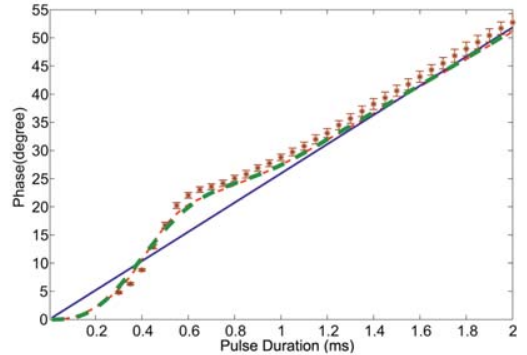

(c)

FIG. 3. Phase difference for different pulse durations for (a) Hard, (b) Fermi, and (c) SLR pulses with a 2-kHz offset frequency.

and a $4-\mathrm{kHz}$ offset-frequency (note that the imaging parameters were set to $150-\mathrm{ms}$ TR, 14.5-ms TE, 5-mm slice thickness, $256 \times 256$ in-plane resolution, and 200mm field of view) and using the linear relation between the induced $B_{1}$ field and the applied voltage, the magnitudes of the $B_{1}$ fields were acquired and the phase shifts obtained at the same points on the phase image were noted for each applied voltage. This experiment was repeated for offset frequencies of $100 \mathrm{~Hz}, 1$ and $4 \mathrm{kHz}$. The experiments were repeated five times for each pulse and offset frequency. The $B_{1}^{+}$versus phase plots were computed with the mean values, and the standard deviations computed across the five repeats.

Before each experiment, the $B_{0}$ offset frequency was minimized using manual shimming. Note that the measured $B_{0}$ offset frequencies after the shimming were taken into account for both the simulations and the $\left|B_{1}^{+}\right|$value calculations with Eqs. [1] and [3].

\section{RESULTS}

\section{Effect of the Pulse Duration}

In Figure 3, we present a comparison of the phase shifts obtained by simulations, by MR experiments, by applying Eq. [1], and by applying Eq. [15] for different pulse durations and for the hard, Fermi, and SLR pulse shapes with a $2-\mathrm{kHz}$ offset frequency. Note that the peak $\left|B_{1}^{+}\right|$ values were estimated to be $12.6 \mu \mathrm{T}$ for the hard pulse, 16.2 $\mu \mathrm{T}$ for the Fermi pulse, and $21.1 \mu \mathrm{T}$ for the SLR pulse, where $\left(\omega_{1} / \omega_{R F}\right) \leq 0.5$. The figure shows that the results of the experiments follow the results of the Bloch simulations. Furthermore, the phase shifts obtained by Eq. [15] and those obtained by the Bloch simulations exhibit a similar behavior in terms of their dependence on the pulse duration. However, there is an appreciable difference between the results of Eq. [1] and the results of the simulations. This difference is more significant for the Fermi and SLR pulses than for the hard pulse. To compare the results quantitatively, the absolute maximum phase differences of the closed form expressions $\left(\phi_{\mathrm{TD}}\right.$ and $\phi_{\mathrm{FD}}$ ) relative to the simulation and experimental results have been calculated. The absolute maximum phase differences between $\phi_{\mathrm{FD}}$ and the Bloch simulations is less than $1^{\circ}$ for all pulse shapes. However, for the hard, Fermi, and SLR pulse shapes, the absolute maximum phase differences between $\phi_{\mathrm{TD}}$ and the Bloch simulations are $2.5^{\circ}, 4^{\circ}$, and $5^{\circ}$ at $0.6-\mathrm{ms}$ pulse duration corresponding to 20,24 , and $25 \%$ errors, respectively. Note that the absolute maximum phase differences between $\phi_{\mathrm{TD}}$ and the experiments are approximately $6^{\circ}$ at the 0.6-ms pulse duration for the Fermi and SLR pulse shapes.

In Figure 4, the $\left|B_{1}\right|$ maps obtained for a Fermi pulse with an 8-ms pulse duration, 4-kHz offset frequency and $0.6-\mathrm{ms}$ pulse duration $2-\mathrm{kHz}$ offset frequency, the $B_{0}$ map, and the difference between the $\left|B_{1}\right|$ maps obtained with the time domain approximation and frequency domain approximation are shown. To obtain each $\left|B_{1}\right|$ map, the $B_{0}$ map given in Figure $4 \mathrm{~d}$ was taken into account. Figure 4a was taken as a reference $\left|B_{1}\right|$ map (Note that for a Fermi pulse with an 8-ms pulse duration and $4-\mathrm{kHz}$ offset frequency, both the time domain approximation and the frequency domain approximation gave the same $\left|B_{1}\right|$ map). To obtain the $\left|B_{1}\right|$ maps given in Figure $4 \mathrm{~b}$ and $\mathrm{c}$, a Fermi pulse with a 0.6 -ms pulse duration and $2-\mathrm{kHz}$ offset frequency was used. Figure $4 \mathrm{~b}$ was obtained using the time domain approximation and Figure 4c was obtained using the frequency domain approximation. It is observed that for the same phase shift, the calculated $\left|B_{1}\right|$ value is much higher than the expected value when the time domain approximation was used. Figure $4 \mathrm{e}$ and $\mathrm{f}$ also show the difference between the reference $\left|B_{1}\right|$ map and the $\left|B_{1}\right|$ maps obtained with the time domain approximation and the frequency domain approximation, respectively.

\section{Effect of the Off-Resonance Frequency}

In Figure 5, we present a comparison of the phase shifts obtained through Bloch simulations, those observed in the experiments, those obtained by Eq. [1], and those obtained by Eq. [15] for different $B_{1}$ magnitudes and offset frequencies. From the applied voltages, the excitation $\mathrm{RF}$ peak $\mid B_{1}^{+}$ | is estimated to be $29 \mu \mathrm{T}$. All results match very closely at 1 and $4 \mathrm{kHz}$ frequencies. However, when the offset frequency is $100 \mathrm{~Hz}$, the results of Eq. [1] start to deviate from the results of the Bloch equations and from the results of experiments, whereas Eq. [15] gives closer results. At low offset frequencies, precise knowledge of the $B_{0}$ field and therefore, the $B_{0}$ frequency offset is critically important. In these experiments, the $B_{0}$ offset frequency was measured to be $25 \mathrm{~Hz}$, and this value was taken into account during the simulations and the calculations.

With the data shown in Figure 5, the percent errors (i.e., $\left.\left|\phi_{n 1}-\phi_{n 2}\right| /\left(\phi_{n 1}\right) \times 100\right)$ between the results of the 


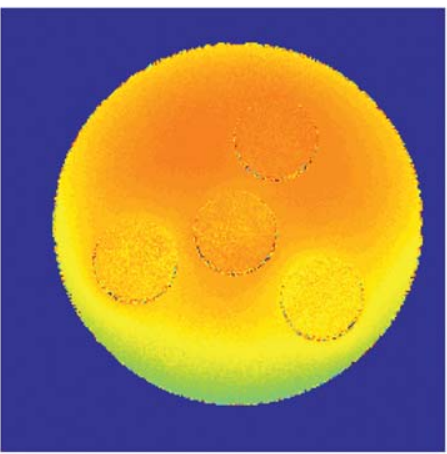

(a)

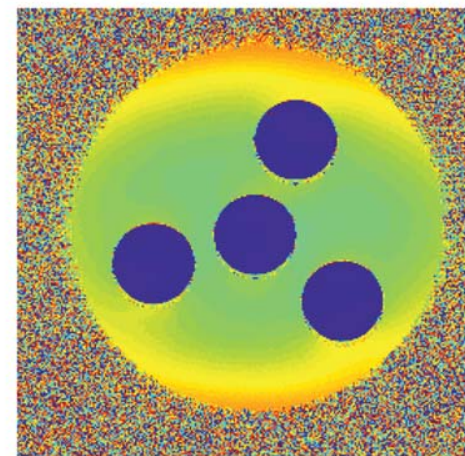

(d)

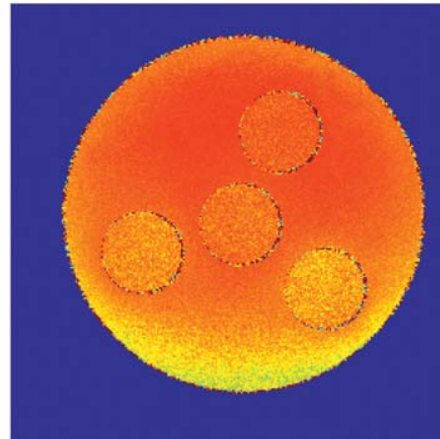

(b)

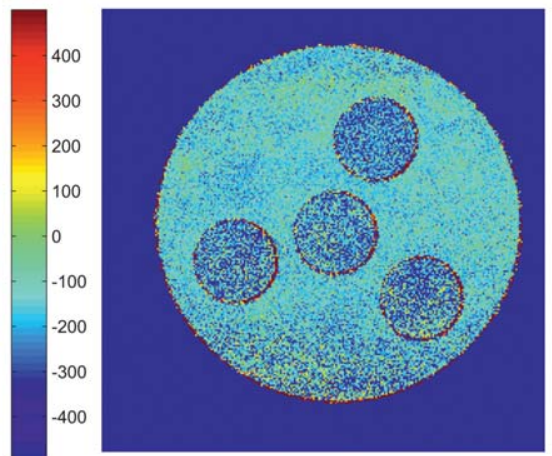

(c)
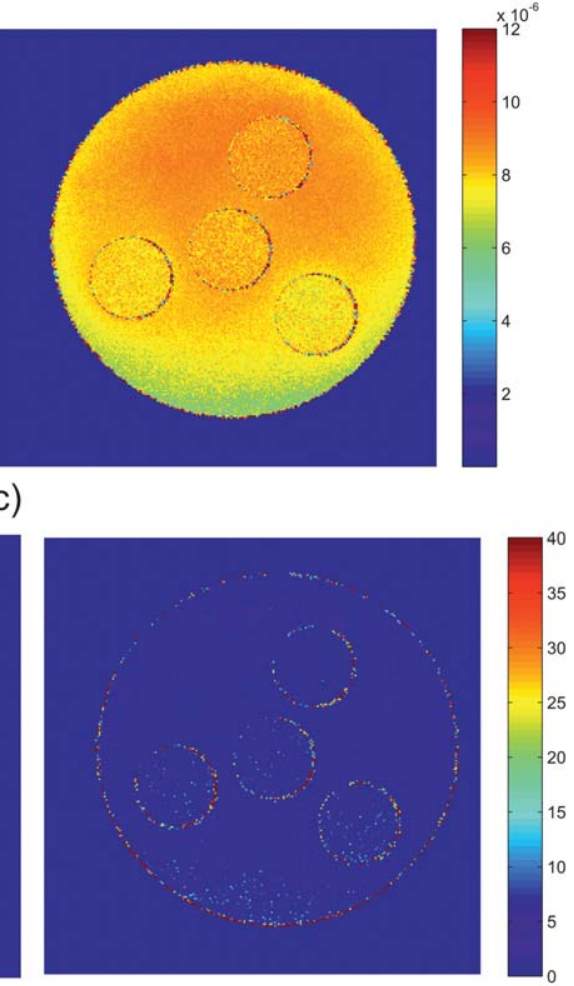

(f)

FIG. 4. $\left|B_{1}\right|$ map (in terms of $T$ ) of the phantom obtained for $60^{\circ}$ flip angle (a) with a Fermi pulse (8-ms pulse duration, 4-kHz offset frequency) and using the $\phi_{\text {TD }}$ expression, (b) with a Fermi pulse (0.6-ms pulse duration, 2-kHz offset frequency) and using the $\phi_{\mathrm{TD}}$ expression, (c) with a Fermi pulse (0.6-ms pulse duration, $2-\mathrm{kHz}$ offset frequency) and using the $\phi_{\mathrm{FD}}$ expression. d: $B_{0}$ map (in terms of degree) obtained with two phase images of gradient echo sequences with TE $=5 \mathrm{~ms}$ and TE $=6 \mathrm{~ms}$, (e) Difference (in terms of \%) of the $\left|B_{1}\right|$ maps obtained in (a) and (b), (f) Difference (in terms of \%) of the $\left|B_{1}\right|$ maps obtained in (a) and (c).

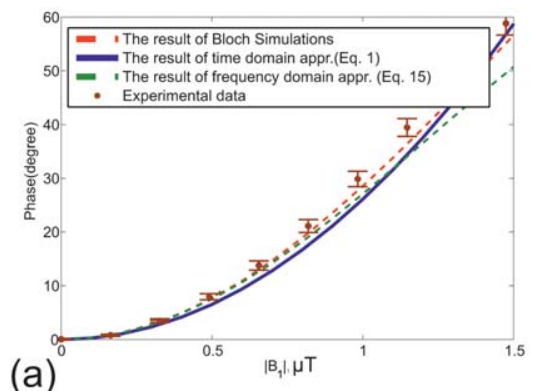

(a)

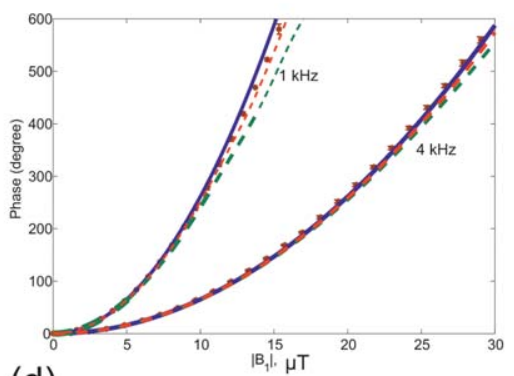

(d)

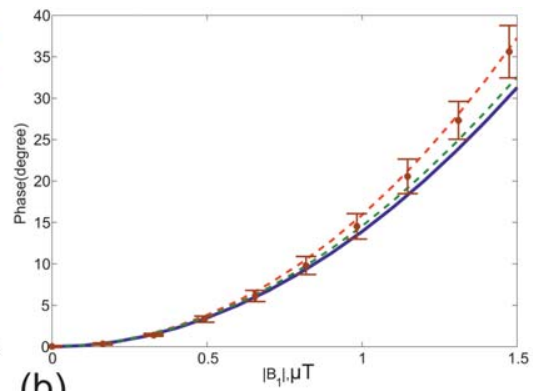

(b)

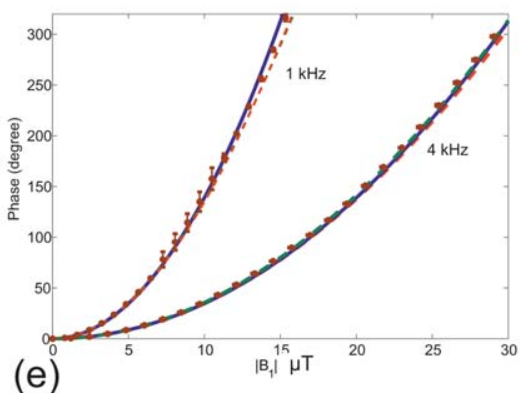

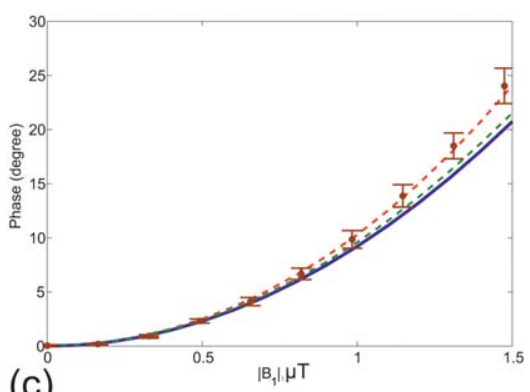

(c)

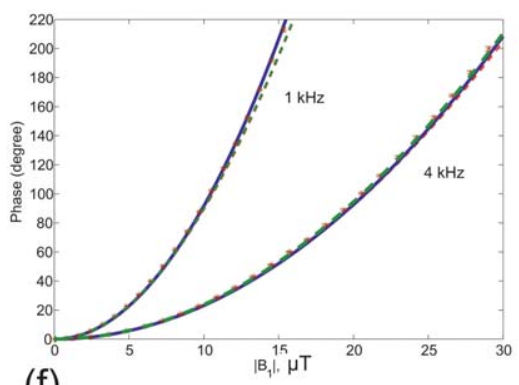

(f)

FIG. 5. Relation of the phase to the magnitude of $B_{1}$ for (a) Hard, (b) Fermi, and (c) SLR pulses with a 100-Hz offset frequency and 8ms pulse duration. Relation of the phase to the magnitude of $B_{1}$ for (d) Hard, (e) Fermi, and (f) SLR pulses with 1 and 4 kHz offset frequencies and 8-ms pulse duration. 


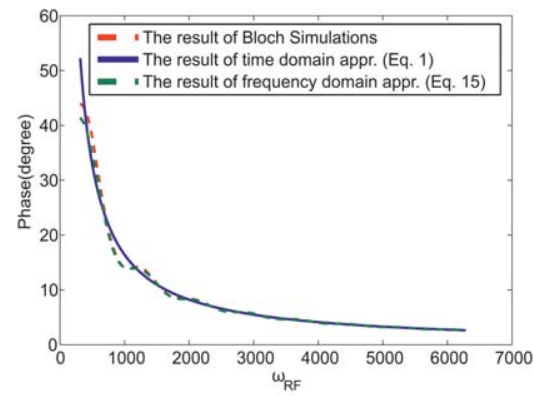

(a)

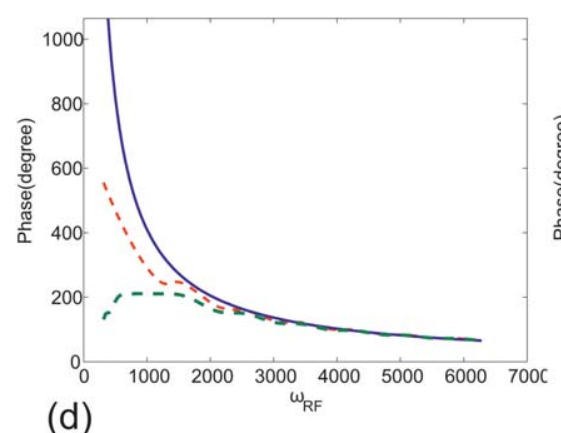

(d)

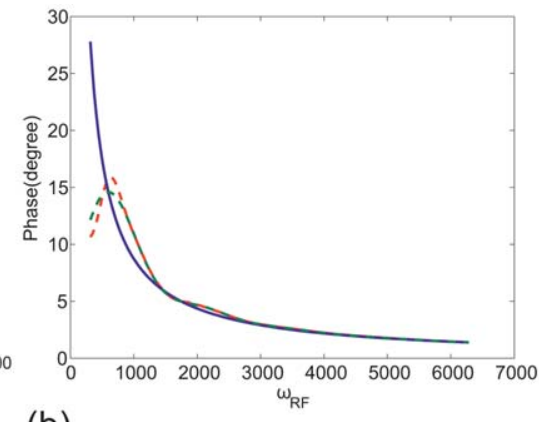

(b)

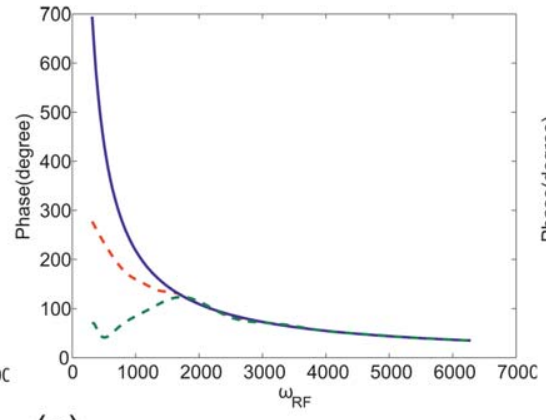

(e)

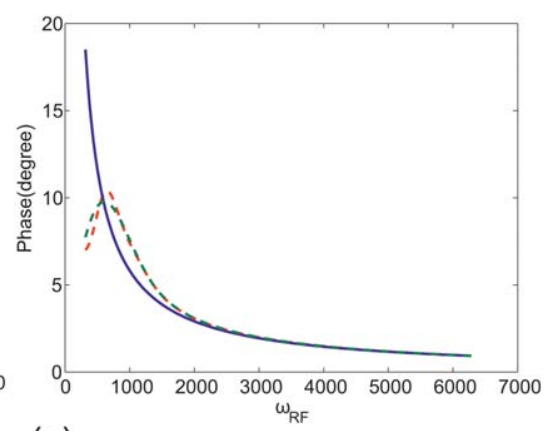

(c)

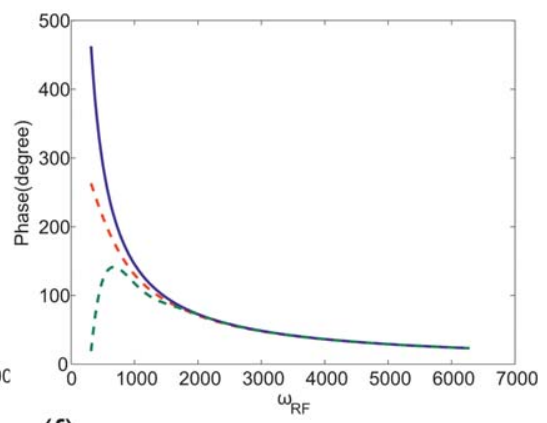

(f)

FIG. 6. Relation of $\omega_{\mathrm{RF}}$ to the phase shift for (a) Hard, (b) Fermi, and (c) SLR pulses with an 8-ms pulse duration and $\left|B_{1}^{+}\right|=1 \mu \mathrm{T}$, (d) Hard, (e) Fermi, and (f) SLR pulses with an 8-ms pulse duration and $\left|B_{1}^{+}\right|=5 \mu \mathrm{T}$.

simulations and the results of Eq. [1] and also between the simulations and the results of Eq. [15] were calculated to investigate the accuracy of the equations in relation to the approximation $\omega_{\mathrm{RF}}>>\omega_{1}$. The error for each pulse shape was calculated to be smaller than $3 \%$ at the $4 \mathrm{kHz}$ offset frequency for $B_{1}$ values up to $29 \mu \mathrm{T}$, for which $\left(\omega_{1} / \omega_{R F}\right) \leq 0.3$ applies. For the hard and Fermi pulse shapes with a $1-\mathrm{kHz}$ offset frequency, the error was smaller than $5 \%$ when $\left(\omega_{1} / \omega_{R F}\right) \leq 0.5$. For the SLR pulse shape with a $1-\mathrm{kHz}$ offset frequency, the error between the results of the simulations and the results of Eq. [1] was smaller than $5 \%$ when $\left(\omega_{1} / \omega_{R F}\right) \leq 0.55$, and the error between the results of the simulations and the results of Eq. [15] was smaller than 5\% when $\left(\omega_{1} / \omega_{R F}\right) \leq 0.62$. For all of the pulse shapes with a $100-$ $\mathrm{Hz}$ offset frequency, the error between the results of the simulations and the results of Eq. [1] was more than $8 \%$, but the error between the results of the simulations and the results of Eq. [15] was less than 5\% when $\left(\omega_{1} / \omega_{\mathrm{RF}}\right) \leq 0.55$.

In Figure 6, we demonstrate the relation between $\omega_{R F}$ and the phase for the hard, Fermi, and SLR pulse shapes with an 8-ms pulse duration and 1 and $5 \mu \mathrm{T}$ peak $B_{1}$ magnitudes. When $1 \mu \mathrm{T}$ is used as the peak $B_{1}$ magnitude, $\omega_{1} / \omega_{\mathrm{RF}} \leq 0.85$, and there is a limitation in reducing the offset frequency to increase the phase. Figure 6a-c shows that the inverse proportionality between the phase and the offset frequency starts to become invalid after some frequency. These figures also show that the results of the frequency domain approximation $\left(\phi_{\mathrm{FD}}\right)$ follow the results of the simulations quite well for all the simulated frequency points for the $1 \mu \mathrm{T}$ peak $B_{1}$ magnitude, even though the time domain approximation $\left(\phi_{\mathrm{TD}}\right)$ fails at lower offset frequencies. In contrast, for the $5 \mu \mathrm{T}$ peak $B_{1}$ magnitude and $\omega_{1} / \omega_{\mathrm{RF}} \leq 4.26$, both the time domain approximation and the frequency domain approximation fail at lower offset frequencies.

\section{DISCUSSION AND CONCLUSIONS}

In this study, we have presented a new approximated Fourier domain expression to increase the understandability of the BS-based $B_{1}$ mapping method. Using this expression, $\left|B_{1}^{+}\right|$, values can be predicted from the phase data using the Fourier transform of the BS RF pulse.

When Plancherel's theorem is used, the time domain approximated expression can be written in a manner similar to the frequency domain approximated expression. When the BS RF pulse has a narrow bandwidth, it can easily be shown that the time domain and the frequency domain approximated expressions are equivalent to each other. Although the expressions are similar, they are not identical. In fact, as shown with simulations, the frequency domain representation is more accurate for BS $\mathrm{RF}$ pulses with wide spectral content such as short RF pulses. This finding is not surprising because the frequency domain expression is formulated based on the phase difference between the actual BS shift and its time-domain expression.

The hard, Fermi, and SLR pulse shapes were used to compare the results of the simulations and the results 
obtained by the time domain approximation and the frequency domain approximation. For each pulse shape, different pulse durations and offset frequencies were used to analyze the effect of each parameter and to verify the frequency domain approximation.

Considering the TE value limitation, the pulse duration was decreased for each pulse shape. When the frequency domain approximation is used, it is observed that the frequency domain relation of the BS pulse shape also affects the relation between the pulse duration and the phase. In contrast, for the time-domain approximation, the duration of the BS pulse and the phase shift appear to have a simple linear relation for a constant offset frequency and a constant $B_{1}$ amplitude.

Note that the decrease in the signal level when the offset frequency is decreased should also be considered. It can be argued that use of low offset frequencies may be counterproductive because the MR signal level may decrease due to on-resonance effects. In our experiments, when the BS pulses with $100-\mathrm{Hz}$ offset frequency were used, the signal level decreased by up to $50 \%$, whereas | $B_{1}^{+} \mid$increased from 0.5 to $5 \mu \mathrm{T}$. In contrast, in the $\left|B_{1}\right|$ range for which $\omega_{1} / \omega_{\mathrm{RF}} \leq 0.5$, there was a $10 \%$ decrease in the signal level. Therefore, when the specific absorption rate limitation becomes the principal concern, the low offset frequency can be decreased under the following condition: $\omega_{1} / \omega_{\mathrm{RF}} \leq 0.5$. Furthermore, in certain cases such as when surface coils are used, the $B_{1}$ profile has different $B_{1}$ values ranging from low to high; to obtain the correct image profiles, small $\left|B_{1}\right|$ values also need to be measured. When using BS pulses with high offset frequencies, the phase-shift for these low-level $\left|B_{1}\right|$ values will be noisy. For the correct calculation of these low-level values, it is beneficial to use BS pulses with low offset frequencies.

During the simulations and experiments crusher gradients were also used, as suggested in (6), and their effects were monitored. Our observations indicate that crusher gradients must be used to minimize the echo originating from on-resonance excitation by the offresonance pulse, especially when low offset frequencies and small pulse durations are used.

In conclusion, simulations and experiments show that the proposed frequency domain approximated expression works well even for short pulse durations and low offset frequencies when the condition $\omega_{1} / \omega_{R F} \leq 0.5$ is valid. Moreover, because the frequency domain expression supplies more information about the relation between the pulse shape and the phase shift, this expression can also be used to design new BS pulse shapes.

\section{APPENDIX A}

Eq. [7] is rewritten as follows:

$$
\begin{gathered}
\frac{\mathrm{d}}{\mathrm{d} t} M_{\mathrm{y}}(t)=\omega_{1 x}(t) M_{z}(t)+\omega_{\mathrm{TD}} M_{0}, \\
\frac{\mathrm{d}}{\mathrm{d} t} M_{z}(t)=-\omega_{1 x}(t) M_{y}(t)+\omega_{1 y}(t) M_{0} .
\end{gathered}
$$

Note that $\omega_{1 x}=\gamma B_{1}^{\mathrm{e}}(t) \cos \left(\int_{0}^{T}\left(\omega_{\mathrm{RF}}(t)-\omega_{\mathrm{TD}}\right) \mathrm{d} t+\theta+\theta_{0}\right)$ and $\omega_{1 y}=\gamma B_{1}^{\mathrm{e}}(t) \sin \left(\int_{0}^{T}\left(\omega_{\mathrm{RF}}(t)-\omega_{\mathrm{TD}}\right) \mathrm{d} t+\theta+\theta_{0}\right)$. These differential equations are written as a single differential equation in the form of $M_{y z}$ where $M_{y z}=M_{y}+i M_{z}$

$$
\frac{\mathrm{d}}{\mathrm{d} t} M_{\mathrm{yz}}(t)=-i \omega_{1 \mathrm{x}}(t) M_{\mathrm{yz}}(t)+\left(\omega_{\mathrm{TD}}+i \omega_{1 y}(t)\right) M_{0} .
$$

The solution to this first order differential equation can be written as:

$$
M_{y z}(t)=f(t) \exp \left(-i \int_{0}^{t} \omega_{1 x}(s) \mathrm{d} s\right) .
$$

To find $f(t)$, this solution is plugged into Eq. [20]. As a result, the solution for $M_{y z}$ at time $T$ is found to be the following:

$$
M_{\mathrm{yz}}(T)=M_{0} \int_{0}^{T}\left(\omega_{\mathrm{TD}}+i \omega_{1 y}(t)\right) \exp \left(-i \int_{t}^{T} \omega_{1 x}(s) \mathrm{d} s\right) \mathrm{d} t .
$$

\section{APPENDIX B}

To find a simplified solution for the $\phi_{\mathrm{BS}}$ given in Eq. [13], the limits of the integration are changed by adding a unit step function $[u(t)]$ as follows:

$$
\phi_{\mathrm{BS}} \approx-\int_{0}^{T} \int_{0}^{T} \omega_{1 y}(t) \omega_{1 x}(s) u(s-t) \mathrm{d} s \mathrm{~d} t
$$

$\omega_{1 x}(t)$ and $\omega_{1 y}(t)$ are expressed in terms of $\omega_{1}(t)$ and $\omega_{1}^{*}(t)$, where $\omega_{1}^{*}(t)$ is the complex conjugate of $\omega_{1}(t)$, and Eq. [23] is rewritten in terms of $\omega_{1}(t)$ and $\omega_{1}(t)^{*}$ as follows:

$$
\phi_{\mathrm{BS}} \approx-\int_{0}^{T} \int_{0}^{T} \frac{\omega_{1}(t)-\omega_{1}^{*}(t)}{2 i} \frac{\omega_{1}(s)+\omega_{1}^{*}(s)}{2} u(s-t) \mathrm{d} s \mathrm{~d} t .
$$

To obtain a Fourier relation instead of an $\omega_{1}(t)$ term, we used the Fourier relation $\int_{-\infty}^{\infty} \Omega_{1}\left(f_{t}\right) \exp \left(i 2 \pi f_{t} t\right) \mathrm{d} f_{t}$ as follows:

$$
\begin{aligned}
\phi_{\mathrm{BS}} \approx-\int_{0}^{T} \int_{0}^{T} \int_{-\infty}^{\infty} \int_{-\infty}^{\infty} \frac{\Omega_{1}\left(f_{t}\right)-\Omega_{1}^{*}\left(-f_{t}\right)}{2 i} e^{i 2 \pi f_{t} t} \\
\frac{\Omega_{1}\left(f_{s}\right)+\Omega_{1}^{*}\left(-f_{s}\right)}{2} e^{i 2 \pi f_{s} s} u(s-t) \mathrm{d} f_{s} \mathrm{~d} f_{t} \mathrm{~d} s \mathrm{~d} t
\end{aligned}
$$

The variables $t$ and $s$ are replaced with the new variables $q$ and $r$, where $s=(r+q) / \sqrt{2}$ and $t=(q-r) / \sqrt{2}$. By changing the order of the integrals and using the relation:

$$
\int_{-\infty}^{\infty} u(\sqrt{2} r) e^{\left(i 2 \pi f_{r} r\right)} \mathrm{d} r=-\left(\frac{1}{2} \delta\left(f_{r}\right)+\frac{1}{i 2 \pi f_{r}}\right)
$$

the final expression becomes the following:

$$
\phi_{\mathrm{BS}} \approx-\int_{-\infty}^{\infty} \frac{\left|\Omega_{1}(f)\right|^{2}}{4 \pi f} d f-\frac{\Omega_{1}^{2}(0)-\Omega_{1}^{* 2}(0)}{8 i} .
$$

Because $\omega_{1}(t)$ is defined in a BSTD rotating frame, $\left(\omega_{0}+\omega_{\mathrm{TD}}\right.$ rotating frame $)$ such as: 


$$
\omega_{1}(t)=\gamma B_{1}^{e}(t) \exp \left[i\left(\int_{0}^{t}\left(\omega_{\mathrm{RF}}(\tau)-\omega_{\mathrm{TD}}\right) \mathrm{d} \tau\right)\right] \exp \left(i\left(\theta+\theta_{0}\right)\right),
$$

the term $e^{i\left(\theta+\theta_{0}\right)}$ stands out in the $\Omega_{1}(f)$ term. The second part of Eq. [27] also includes these phase terms. In contrast, because the phase difference of two acquisitions taken with positive and negative offset frequencies is used and the term $e^{i\left(\theta+\theta_{0}\right)}$ does not change, we can ignore this part. Thus, the expression simplifies to the following relation:

$$
\phi_{\mathrm{BS}} \approx-\int_{-\infty}^{\infty} \frac{\left|\Omega_{1}(f)\right|^{2}}{4 \pi f} \mathrm{~d} f
$$

\section{REFERENCES}

1. Sacolick LI, Wiesinger F, Hancu I, Vogel MW. $B_{1}$ mapping by BlochSiegert shift. Magn Reson Med 2010;63:1315-1322.

2. Ramsey NF. Resonance transitions induced by perturbations at two or more different frequencies. Phys Rev 1955;100:1191-1194.

3. Khalighi MM, Rutt BK, Kerr AB. RF pulse optimization for BlochSiegert $B_{1}^{+}$mapping. Magn Reson Med 2012;68:857-862.
4. Jankiewicz M, Gore JC, Grissom WA. Improved encoding pulses for Bloch-Siegert $B_{1}^{+}$mapping. J Magn Reson 2013;226:79-87.

5. Khalighi MM, Rutt BK, Kerr AB. Adiabatic RF pulse design for Bloch-Siegert $B_{1}^{+}$mapping. Magn Reson Med 2013;70:829-835.

6. Duan Q, van Gelderen P, Duyn J. Improved Bloch-Siegert based B1 mapping by reducing off-resonance shift. NMR Biomed 2013;26: 1070-1078.

7. Basse-Lüsebrink TC, Kampf T, Fischer A, Sturm VJF, Neumann D, Köstler H, Hahn D, Stoll G, Jakob PM. SAR-reduced spin-echo-based Bloch-Siegert B1+ mapping: BS-SE-BURST. Magn Reson Med 2012; 68:529-536.

8. Saranathan M, Khalighi MM, Glover GH, Pandit P, Rutt BK, Efficient Bloch-Siegert $B_{1}^{+}$mapping using spiral and echo-planar readouts. Magn Reson Med 2013;70:1669-1673.

9. Basse-Lüsebrink, TC, Sturm VJF, Kampf T, Stoll G, Jakob PM. Fast CPMG-based Bloch-Siegert B1 mapping. Magn Reson Med 2012;67: 405-418.

10. Bloch F, Siegert A. Magnetic resonance for nonrotating fields. Phys Rev 1940;57:522-527.

11. Turk EA, Ider YZ, Atalar E. Analysis of B1 mapping by Bloch Siegert shift. In Proceedings of the 14th Annual Meeting of ISMRM, Melbourne, Australia, 2012. p. 608.

12. Gerald BM. An integrated program for amplitude-modulated RF pulse generation and re-mapping with shaped gradients. Magn Reson Imaging 1994;12:1205-1225.

13. Ghiglia DC, Pritt MD. Two-dimensional phase unwrapping: Theory, algorithms, and software. New York: Wiley Interscience; 1998. 